\title{
Evaluación de sitios web de postgrados biomédicos en España
}

\author{
Evaluation of websites for biomedical \\ postgraduate courses in Spanish
}

\author{
María-Dolores OLVERA-LOBO',2 \\ María AGUILAR-SOTO3 \\ Elvira RUIZ-DE-OSMA ${ }^{1}$
}

\section{Resumen}

El objeto de este trabajo es la creación de una herramienta para la evaluación de la calidad de la información contenida en los sitios web de Postgrado de ámbito biosanitario en las universidades españolas. Se ha diseñado y desarrollado una hoja de evaluación (checklist) que ha sido validada y aplicada a los 131 sitios web de Postgrado con Mención de Calidad de tema biosanitario de las universidades españolas. Se han analizado las valoraciones obtenidas por los sitios web y se han aplicado técnicas de clustering y de análisis de componentes principales. Los datos recogidos por la checklist permiten establecer un ranking según la calidad de la información de los sitios web. Además, se observa la existencia de tres grandes grupos de sitios web según sus características y prestaciones. La aplicación de las herramientas diseñadas indica que los sitios web alcanzan valores aceptables, si bien presentan algunos defectos comunes. No obstante, se constata la existencia de varios niveles de calidad de los mismos.

Palabras-clave: Enseñanzas de postgrado. Evaluación de sitios web. Universidades españolas.

\begin{abstract}
The aim of this work is to create a tool for assessing the quality of the information on postgraduate course websites at Spanish universities. An evaluation checklist was developed and applied to the 131 websites of postgraduate biomedical courses with quality accreditation in Spanish universities. The website evaluations were analysed with the application of clustering and principal component analysis techniques. While the average of all the sites is 'acceptable' there remain some clear weaknesses in aspects such as accessibility, lack of an internal search engine, or forms - for obtaining the views of current students and lecturers-and evaluation tests - for analysing the results. The tool developed provides a new instrument for evaluating postgraduate course websites. This evaluation enables website comparison, helps identify their strengths and weaknesses, and facilitates their improvement.
\end{abstract}

Keywords: Postgraduate courses. Website evaluation. Spanish universities.

\section{Introducción}

Los actuales Másteres Universitarios son la primera muestra de la nueva configuración de los estudios universitarios en el marco del Espacio Europeo de Educación Superior. Su objetivo es proporcionar al estudiante una formación más avanzada, especializada y multidisplicinar. Sus objetivos son tanto el

\footnotetext{
1 Profesora Doctora, Universidad de Granada, Facultad de Comunicación y Documentación, Departamento de Biblioteconomía y Documentación. Campus de Cartuja, 18071, Granada, España. Correspondencia a nombre de/Correspondence to: M.D. OLVERA-LOBO. E-mail: <molvera@ugr.es>.

2 Consejo Superior de Investigaciones Científicas, Unidad Asociada Grupo SCImago. Madrid, Espanã.

3 Becaria investigadora, Universidad de Granada, Facultad de Comunicación y Documentación, Departamento de Biblioteconomía y Documentación. Granada, España.

Recebido el día 7/11/2011 y aceptado para su publicación el 17/11/2011.
} 
perfeccionamiento de la esfera profesional como la investigación. Estos estudios se estructuran en Máster y Doctorado (España, 2007), de manera que la superación de estas enseñanzas da lugar a los títulos de Máster Universitario y de Doctor o Doctora, respectivamente.

La convergencia europea conlleva la adopción de una serie de medidas en la Universidad española, una de las cuales fue la creación de la Mención de Calidad para los programas de doctorado que ofertaban y que cumplían una serie de requisitos de calidad, tanto en sus contenidos como en su estructura y objetivos (Buela-Casal; Castro, 2008).

Este trabajo se centra en el desarrollo de una herramienta para la evaluación de los sitios web de los POP de las universidades españolas. Habida cuenta de que la mayoría de las Universidades contemplan en sus estudios de Postgrado tanto Másteres como Doctorados especializados en algún aspecto de la Biomedicina, la checklist se ha aplicado a los sitios web biomédicos que cuentan con Mención de Calidad, los cuales, en el curso 2008-2009, ascendían a 131. En general, la temática tratada en estos cursos es muy amplia y abarca disciplinas como Biotecnología, Bioquímica, Salud mental, Genética, Microbiología, Inmunología, Psicología, Medicina Interna o Fisiología, entre otras, aunque se observa un mayor interés en los temas de Biología Celular, Biología Molecular, Nutrición, Metabolismo, Desarrollo e Innovación de Alimentos, Farmacología, Desarrollo y Uso Racional de Medicamentos y Neurociencia.

La universidad que ofrece mayor número de Postgrados es la Universidad de Barcelona, con un total de 22. Le sigue Granada con 18 cursos, la Universidad Autónoma de Cataluña con 15, la Universidad Complutense y la de Navarra con 7 Postgrados, la Universidad de La Coruña con 6 y la Autónoma de Madrid con 5.

\section{Métodos}

La recopilación de las direcciones web de los Postgrados biomédicos con Mención de Calidad de las Universidades españolas se efectuó a partir del sitio web del Ministerio de Educación de España. Algunos Postgrados cuentan con un sitio web independiente, aunque su URL esté bajo el dominio de la universidad, y otros se encuentran alojados en el sitio web genérico de su universidad.
En la realización de este trabajo se ha aplicado una herramienta en forma de hoja de evaluación o cheklist. Se asegura su validez interna con un alfa de Cronbach de 0,81. La checklist se basa en criterios aplicados por diferentes autores, si bien se han incluido indicadores específicamente adaptados a las necesidades concretas del objeto deestudio. Además se han utilizando herramientas como Weblink Validator, W3C Markup Validator y TAW. Durante la revisión de la literatura especializada se observa que cada autor elabora su propio conjunto de indicadores de manera que, aunque se detecta cierta coincidencia, en general no hay homogeneidad.

La checklist se organiza en torno a criterios subdivididos en categorías e incluye un tercer nivel de especificidad, los indicadores (Anexo I).

\section{Checklist}

La checklist aquí creada incorpora los criterios que, tras la revisión bibliográfica, pueden considerarse como básicos para la evaluación de páginas web. Asimismo, se tienen en cuenta otros criterios especialmente adecuados y adaptados al tipo específico de los sitios web que se pretenden evaluar. La fiabilidad de la checklist se ha medido con el test alfa de Cronbach, con una puntuación de 0.8 , por lo que queda demostrada su consistencia interna.

\section{Criterios de carácter general}

Es primordial que en el mundo académico se transmita una imagen de confianza y fiabilidad y que se identifique claramente la universidad a la que está ligada la información, avalando así la calidad del contenido.

\section{Usabilidad y accesibilidad}

La mejor manera de asegurar la usabilidad es tener en cuenta tanto el contexto del uso como las características del usuario desde los primeros procesos del diseño (Wang; Liu, 2007).

Por su parte, la accesibilidad se refiere al acceso universal a la Web, independientemente del tipo de hardware, software, infraestructura de red, idioma, 
localización geográfica y capacidades de los usuarios (Agencia Nacional de Evaluación de la Calidad y Acreditación, 2008).

Asimismo, es necesario tener en cuenta discapacidades asociadas con la edad, la visión, la audición, el habla, la discapacidad motora o la deficiencia cognitiva (Nielsen, 2004). Atender a esta realidad evita en gran medida la exclusión digital.

Para ello, es indispensable asegurarse de que el sitio web supera, al menos, el primero de los tres niveles de la Web Content Accesibility Guidelines (WCAG) de W3C (Panopoulou et al., 2008), que corresponde al primer nivel de conformidad A, aunque lo deseable es que alcance el tercer nivel y el más completo, el triple A.

Relacionados con la usabilidad y la accesibilidad están los aspectos referidos a la navegación, el estilo gráfico y la visibilidad.

La navegación tiene en cuenta las capacidades organizativas y técnicas del proceso de movimiento en y entre las páginas del sitio web (Merwe; Bekker, 2003), y está relacionada con la funcionalidad de los sitios web y la facilidad de uso (Olsina et al., 2008), el contenido, la estructura física, el control y la capacidad para adaptarlo a los gustos del usuario. También se ocupa de los elementos de presentación (Oppenheim; Ward, 2006), de la consistencia y de la organización. Para aprovechar la capacidad hipertextual de la Web existen otros aspectos significativos, como la estructura lógica del sitio ?(Merwe; Bekker, 2003), la sencillez del mismo (Djajadikerta; Triredsani, 2006) y los enlaces que incluye (Nielsen, 2004; Oppenheim; Ward, 2006).

Por su parte, el estilo gráfico se refiere a los aspectos visuales del sitio web (Djajadikerta; Trireksani, 2006) - cuán atractivo es visualmente - y al uso de las fuentes, colores y fondo.

\section{Informacion y contenidos}

Una vez atraído el usuario al sitio web hay que ocuparse del contenido (Brock; Zhou, 2005; Barnes; Vidget, 2007) así como de su calidad (Djajadikerta; Trireksani, 2006). La calidad del contenido dependerá de la relevancia de la información para el usuario (Miranda González; Bañegil Palacios, 2004) y de su cantidad (Merwe; Bekker, 2003).
Como criterios de contenido adecuado y comprensible, se encuentran la credibilidad, la claridad de la información y la concisión. No hay que perder de vista la originalidad de la información, es decir, que ésta no pueda localizarse en otros sites (Jiménez Piano; OrtizRepiso Jiménez, 2007).

En definitiva, la información debe ser completa, actualizada, real, detallada, exacta y comprensible.

Nuestra cheklist incluye algunos indicadores para evaluar la información característica de estos sitios, es decir, aquello que tiene que ver con los programas de estudio, créditos, metodología, condiciones de acceso etc.

En relación a la claridad, hay que decir que son muchas las características deseables de la información para que ésta tenga "valor" (Toro, 2002), pero resulta fundamental que la formulación esté acorde con la audiencia a la que se dirige (Smith, 2001; Jiménez Piano; Ortiz-Repiso Jiménez, 2007). Mencionaremos la objetividad esperada de la información, su organización y aspectos como el buen uso del lenguaje, la concisión y la falta de errores (Smith 2001; Merwe; Bekker, 2003; Jiménez Piano; Ortiz-Repiso Jiménez, 2007). Para la evaluación de sitios web se pueden considerar además las lenguas diferentes en las que se encuentra el texto (Holzer; Kim, 2005; Henriksson et al., 2006) así como la extensión de texto que ha sido traducido a cada idioma (Panopoulou et al., 2008).

La categoría requisitos y resultados se refiere tanto a la información de ingreso y a los requisitos para acceder a los estudios de postgrado (Olsina et al., 2008) como a los logros de los estudiantes - indicador también usado por la británica Quality Assurance Agency for Higher Education. Se trata de evaluar si la información referente a estos aspectos es suficiente. Los resultados pueden evaluarse a partir del aporte de los programas de postgrado al desarrollo científico reflejado en el sitio web mediante la relación de publicaciones, tesis y tesinas realizadas y en proyecto. Otros indicadores son también la información sobre la inserción laboral de los titulados y sus resultados académicos.

La categoría formularios y test de valoración en la evaluación de estos sitios web se justifica por la importancia de establecer mecanismos de seguimiento de los resultados de los programas de POP, especialmente 
en lo relativo a la opinión de estudiantes y doctores egresados, y tenerla en cuenta para la mejora del programa (Agencia Nacional de Evaluación de la Calidad y Acreditación, 2008).

\section{Servicios}

Aquí se han analizado aspectos relativos al motor de búsqueda interno del sitio web y a las posibilidades de contactar con el personal vinculado al programa académico.

Las utilidades del motor de búsqueda interno suponen un valor añadido (Jiménez Piano; Ortiz-Repiso Jiménez, 2007), ya que son muy utilizados como punto de referencia (Intdev, 2007) y permiten al visitante localizar fácilmente la información específica que está buscando en el sitio web (Miranda Jiménez; Bañegil Palacios, 2004; Brock; Zhou, 2005) de una manera rápida y fácil. Una función de búsqueda global en la página principal ayuda de manera efectiva a la búsqueda de la información y evita la búsqueda navegacional. De todas formas, ambas funciones pueden ser complementarias (Olsina et al., 2008).

El adecuado funcionamiento de las opciones de contactos repercute de manera positiva en la completitud del contenido (Merwe; Bekker, 2003). La importancia de una buena comunicación bidireccional reside en que permite un feedback con el usuario, obteniendo así más información sobre éste (Miranda González; Bañegil Palacios, 2004) y, además, desde su punto de vista (Schubert; Selz, 1999). Esta interactividad mide la disponibilidad de servicios complementarios a la manera tradicional de comunicación en el entorno digital (Buyukozkan et al., 2007).

\section{Ponderación}

Para la checklist se ha utilizado una escala Likert con 4 niveles de respuesta, donde 0 indica ausencia de cierta característica; 1 significa aparece o se menciona; 2 indica que la cumple parcialmente y 3 que la cumple totalmente.

No todos los aspectos de los sitios web tienen la misma importancia para su evaluación. Cada uno es más o menos relevante según el objetivo del sitio.
Las publicaciones especializadas - sobre comercio electrónico, evaluación de sitios web, evaluación de plataformas e-learning, estudios sobre sitios web de universidades o de autoridades públicas - incluyen ponderaciones distintas, atendiendo a los objetivos y características de cada sitio web y otorgando a cada categoría y criterio distinto peso.

Para la ponderación de las categorías, aquí se ha aplicado la propuesta de Panopoulou et al. (2008) como se indica en la Tabla 1.

Los contenidos cuentan con el 50,0\% del peso en la valoración total (Miranda Jiménez; Bañegil Palacios, 2004; Olsina et al., 2008), lo cual responde a la importancia de la información como activo en la toma de decisiones del usuario habida cuenta de cuáles son los objetivos del sitio web de un Postgrado.

El criterio usabilidad y accesibilidad representa el $20,0 \%$, puesto que ya se ha mencionado que los sitios web son las ventanas abiertas de las universidades al mundo exterior (Kutluca et al., 2009). Al fin y al cabo la información puede ser de calidad, pero es necesario que se pueda acceder a ella y esté organizada de la manera que le resulte más adecuada al usuario.

La idea de que el sitio web tenga algún método de feedback a través de la interacción con el usuario

Tabla 1. Ponderaciones de los criterios y categorias.

\begin{tabular}{lcc}
\hline Ponderaciones & \% Categorias & \% Criterios ${ }^{2}$ \\
\hline Características generales & 100 & 10 \\
Usabilidad y accesibilidad & & \\
Navegación & 30 & 20 \\
Estilo gráfico & 20 & \\
Accesibilidad & 25 & 50 \\
Visibilidad & 25 & \\
Información y contenidos & & \\
Investigación & 25 & \\
Requisitos y resultados & 25 & \\
Claridad de La información & 10 & \\
Contenidos & 25 & \\
Formularios/test devaloración & 15 & \\
Servicios & & \\
Motor de búsqueda & 60 & \\
Contactos y accesos & & \\
\hline 1 \% Peso de cada categoria dentro de su criterio; ${ }^{2} \%$ peso de cada criterio \\
en la valoración global del sitio web. \\
Fuente: Panopoulou et al. (2008).
\end{tabular}


refuerza que los contactos y accesos ocupen el 60,0\% del 20,0\% total que supone la categoría Servicios.

\section{Análisis de datos}

A partir de los datos recopilados por la checklist se ha analizado la valoración obtenida por cada sitio web, estableciéndose un ranking de universidades en relación a los indicadores de calidad aplicados a sus sitios web de Postgrados biomédicos con Mención de Calidad.

La correlación entre los criterios y categorías se ha realizado con el procedimiento de correlación bivariado, coeficiente de Spearman y prueba de significación bilateral $(p>0,01)$.

Se ha llevado a cabo un análisis de cluster jerárquico con el método Ward y un análisis de componentes principales con 3 componentes, rotación Varimax y normalización Kaiser.

\section{Resultados}

En general, todos los sitios web tienen un grado aceptable de corrección a nivel lógico, estético e informativo, aunque no por ello están faltos de deficiencias comunes. En este equilibrio encontramos distintos grupos, heterogéneos entre sí y homogéneos como conjunto individual, agrupados según características similares.

Las correlaciones encontradas entre los criterios y categorías de la checklist demuestran que las variables son bastante independientes entre sí (la correlación mayor es de 0,42). Encontramos estas relaciones en los criterios Características Generales y Usabilidad y Accesibilidad, entre Características Generales e Información y Contenidos y entre el criterio Servicios e Información y Contenidos.

El análisis de las puntuaciones obtenidas por los sitios web de POP analizados muestra que el valor medio total obtenido es de 1,5. Esto supone un valor medio entre 1 y 3, lo cual deja claras sus deficiencias generales.

En ninguno de los criterios, a excepción de Características Generales, se alcanza un valor realmente alto o aceptable $(2,43)$. En el caso de Usabilidad y Accesibilidad, las categorías Navegación $(1,98)$ y Estilo Gráfico $(1,65)$ obtienen mejores puntuaciones que las otras dos categorías, Accesibilidad $(0,01)$ y Visibilidad $(1,14)$. El criterio Información y Contenidos consigue un valor algo más alto que Usabilidad y Accesibilidad. La categoría Claridad de la Información obtiene una puntuación más que aceptable $(2,48)$, destacándose del resto de las categorías de su criterio y de los demás criterios. El resto de categorías de este criterio, Investigación $(1,43)$, Requisitos y Resultados $(1,51)$ y Contenidos $(1,83)$ consigue buenas puntuaciones, mientras que Formularios/Test de Valoración obtiene una puntuación bastante deficiente $(0,17)$, ya que la mayoría de las páginas carecían de esta característica. La categoría Servicios adquiere un valor bajo debido a las deficiencias mostradas por las páginas en lo referente al Motor de Búsqueda $(0,17)$ puesto que la mayoría de ellas carece de esta herramienta; sin embargo, la categoría Contactos y Accesos obtiene un valor alto $(1,92)$.

El análisis de cluster y el análisis de componentes principales se apoyan, puesto que en el cluster 1 la mayor adscripción es al componente 2, el cluster 2 al componente 3; y el cluster 3 se adscribe mayoritariamente al componente 1 (Anexo II).

\section{Análisis de cluster}

En el dendrograma generado se observan tres grupos claramente diferenciados. Al realizar la comparación de medias con ANOVA y Bonferroni para permutar entre los tres cluster encontramos diferencias significativas $(p>0,05)$ entre el cluster 1 y $2(-0,17)$ y entre el 1 y $3(-0,43)$, así como entre las medias de los cluster 2 y $3(-0,25)$. Sin embargo, es importante localizar y definir cuáles son estas diferencias para caracterizar los cluster en los que se incluyen los sitios web.

\section{Primer cluster}

El primer grupo abarca 56 sitios web. Dentro de este grupo encontramos los sites que, en conjunto, han obtenido menor puntuación en esta evaluación. La media de todo este conjunto de sitios es de 1,46. Este cluster viene definido por tener promedios altos en el criterio Características Generales y el valor más bajo en Servicios, donde la categoría Motor de Búsqueda presenta un valor 
muy por debajo de lo aceptable. Sin embargo, dentro de este criterio, Contactos y Accesos presenta un valor medio alto, rozando el 2, que podríamos considerarlo muy aceptable. El criterio Usabilidad y Accesibilidad no tiene buen valor general, pero la categoría Navegación consigue un valor cercano al 2, por lo que se considera bastante razonable puesto que es un promedio alto, así como Estilo Gráfico, cuyo valor también es medio-alto. Sin embargo, las categorías Visibilidad y Accesibilidad obtienen la puntuación más baja. El valor del criterio Información y Contenidos es el más bajo de los tres cluster, aunque su categoría Claridad de la Información consigue una puntuación cercana al 2,5, considerada bastante buena, así como Requisitos y Resultados e Investigación. Las categorías con más baja puntuación dentro de este criterio son Contenidos y Formularios/Test de Valoración, que no alcanzan valores medios.

En resumen, sólo el criterio Características Generales presenta un valor razonable, así como las categorías Claridad de la Información, Navegación, Contactos y Accesos y Estilo Gráfico (Tabla 2).

\section{Segundo cluster}

Los 23 sitios web incluidos en este grupo han obtenido la puntuación más alta, aunque algunas características no obtengan valores medios. La media de todos los sitios es de 1,53. Los mejores niveles, como en el cluster anterior, se sitúan en el criterio Características Generales y los valores más bajos en el criterio Servicios, cuya categoría Motor de Búsqueda consigue la puntuación más baja (cero); sin embargo, Contactos y Accesos obtiene un valor cercano al 2, que podemos considerar muy aceptable. El criterio Usabilidad y Accesibilidad se aproxima al valor medio, aunque no lo alcanza. Dentro de este criterio, la categoría Navegación logra un valor superior a 2, que puede considerarse conveniente, así como las categorías Estilo Gráfico y Visibilidad, cuyos valores se pueden estimar aceptables. El peor valor lo tiene la categoría Accesibilidad. El criterio Información y Contenidos tiene un valor medio que se puede considerar apto, y dentro de él encontramos las categorías que más se destacan de los tres cluster y que corresponden a Claridad de la Información, Contenidos; y Requisitos y Resultados, valores que pueden considerarse óptimos. En cambio, las categorías Investigación y, sobre todo, Formularios/Test de Valoración no alcanzan valores estimables.

En resumen, los mejores valores se agrupan en el criterio Características Generales y en las categorías Claridad de la Información, Contenidos y Navegación (Tabla 2).

Tabla 2. Valores de los criterios y categorías en los cluster.

\begin{tabular}{lcccc}
\hline Cluster & 1 & 2 & 3 & POP biomédicos \\
\hline Características generales & 2,33 & 2,65 & 2,45 & 2,43 \\
Identidad & 2,33 & 2,65 & 2,45 & 2,43 \\
Usabilidad y accesibilidad & 1,32 & 1,40 & 1,35 & 1,21 \\
Navegación & 1,91 & 2,14 & 1,97 & 1,98 \\
Estilo gráfico & 1,56 & 1,70 & 1,72 & 1,65 \\
Accesibilidad & 0,02 & 0,00 & 0,00 & 1,14 \\
Visibilidad & 1,10 & 1,26 & 1,13 & 1,47 \\
Información y contenidos & 1,33 & 1,44 & 1,69 & 1,43 \\
Investigación & 1,23 & 0,20 & 2,18 & 2,48 \\
Requisitos y resultados & 1,41 & 1,67 & 2,44 & 1,83 \\
Claridad de la información & 2,46 & 2,66 & 0,17 \\
Contenidos & 1,01 & 2,55 & 0,41 & 1,21 \\
Formularios/test de valoración & 0,09 & 0,04 & 1,48 & 0,17 \\
Servicios & 1,26 & 1,30 & 0,38 & 1,92 \\
Motor de búsqueda & 0,06 & 0,00 & 2,15 & 1,53 \\
Contactos y accesos & 1,72 & 1,86 & 1,60 & \\
Promedio total & 1,46 & 1,53 & & \\
\hline
\end{tabular}

Fuente: Realizada por las autoras. 


\section{Tercer cluster}

Los 52 sitios web que aparecen en este tercer cluster tienen una puntuación media de 1,60, la más alta de los tres.

El criterio que ha obtenido la puntuación más baja es Usabilidad y Accesibilidad. No obstante sus características Navegación y Estilo Gráfico tienen una valoración alta, la característica Visibilidad no consigue llegar al aprobado aunque sobrepasa el 1. La característica peor valorada es Accesibilidad que, al igual que en el segundo cluster, obtiene un cero. El criterio mejor valorado es Características Generales, como en el resto de los cluster. Le sigue en puntuación el criterio Informacióny Contenidos, cuya media es la mejor de los tres cluster, bastante aceptable. Las características mejor valoradas son Claridad de la Información, Contenidos e Investigación. Las tres superan el 2, por lo que podemos decir que son más que aceptables. Requisitos y Resultados obtiene un valor medio y Formularios/Test de Valoración resulta ser la peor valorada, con un deficiente 0,30. El criterio Servicios consigue una puntuación media-baja, aunque es la más alta de los tres cluster. Su característica con mayor promedio es Contactos y Accesos, mientras que Motor de Búsqueda, como ocurre en los otros dos cluster, obtiene una deficiente puntuación de 0,38 (Tabla 2).

\section{Discusión}

A la vista de los resultados se podrían indicar ciertas posibilidades de mejora para estos sitios web.

La información ofrecida al usuario tiene que estar constantemente actualizada, y es necesario que aparezca la fecha de actualización. La existencia de un motor de búsqueda propio facilita la localización de información. Asimismo, es deseable que cuente con una herramienta de traducción a otros idiomas para tener mayor proyección y atraer a una variedad más amplia de estudiantes.

Sería recomendable que apareciese un enlace a la página de créditos o que, al menos, fuera posible tener información acerca del webmaster. Un mapa del sitio también es un elemento muy útil para situar al usuario en toda la página. La ruta de navegación lo orienta a través de los diferentes apartados, por lo que es importante disponer de ella.

Deberían tenerse en cuenta las necesidades especiales de los usuarios y ofrecer la posibilidad de leer la información de la página en letra de mayor tamaño, que se pudiese escuchar el texto o venir apoyado por un vídeo. No atender estas necesidades es perder usuarios potenciales y futuros estudiantes, así como aumentar la brecha digital al imposibilitar su acceso. Hay que cumplir; al menos con el más bajo de los niveles de conformidad a, doble a y triple a.

Sería recomendable que las páginas tuvieran Intranet para permitir la comunicación entre los alumnos que cursan los estudios y los profesores, de modo que la página no solo sea una herramienta informativa.

Requisitos y resultados, perteneciente al criterio Información y Contenidos, es otra de las categorías más deficientes. Sería necesario que los criterios sobre la convalidación de otras titulaciones estuviesen más visibles; también es importante que aparezca información sobre los resultados de las investigaciones llevadas a cabo y las tesis leídas. Esto aporta valor añadido a la información sobre el trabajo de investigación que se desarrolla e indica la dinámica existente en el campo de estudio.

Es muy recomendable la existencia de formularios/test de valoración para que los usuarios potenciales puedan ver la continuidad del propio programa de POP y que los que ya lo hayan cursado mantengan un vínculo con éste a través de la página web.

\section{Conclusiones}

Se ha diseñado y desarrollado una herramienta para la evaluación de sitios web de Postgrado adaptado a las características de las universidades españolas. Esta checklist se ha aplicado a los sitios web de POP biomédicos con Mención de Calidad en las universidades españolas, los cuales, a un nivel general, se puede afirmar que presentan un grado de calidad aceptable. No obstante, todos ellos arrastran algunos defectos comunes en algunos de los criterios y categorías como son, en este caso, la accesibilidad, la visibilidad, el motor de búsqueda y los formularios. 


\section{Referencias}

AGENCIA NACIONAL DE EVALUACIÓN DE LA CALIDAD Y ACREDITACIÓN. Protocolo para la revisión de la página Web. Madrid: Agencia Nacional de Evaluación de La Calidad y Acreditación, 2008. Disponible en: <http://www.aneca.es>. Acceso: 20 jun. 2009.

BARNES, S.J.; VIDGEN, R. Interactive e-government: evaluating the web site of the UK Inland Revenue. International Journal of Electronic Government Research, v.3, n.1, p.19-38, 2007.

BROCK, J.K.U.; ZHOU, Y. Organizational use of the internet: scale development and validation. Interner Reseach, v.15, n.1, p.67-87, 2005.

BUELA-CASAL, G.; CASTRO, A. Análisis de la evolución de los programas de doctorado con mención de calidad en las universidades españolas y pautas para su mejora. Revista de Investigación en Educación, v.5, n.5, p.49-60, 2008.

BUYUKOZKAN, G.; RUAN, D.; FEYZIOGLU, O. Evaluating e-learning web site quality in a fuzzy environment. International Journal of Intelligent Systems, v.22, n.5, p.567-586, 2007

DJAJADIKERTA, H.; TRIRESKSANI, T. Measuring university web site quality: a development of a user-perceiver instrument and its initial implementation to websites of accounting departments in New Zeland's Universities. School of Accounting, Finance and Economics \& FIMARC Working Paper Series, p.1-23, 2006.

ESPAÑA. Real Decreto 1393, de 29 de octubre de 2007, por el que se establece la ordenación de las enseñanzas universitarias oficiales. Boletín Oficial del Estado, n.260, 30 oct. 2007.

HENRIKSSON, A. et al. Evaluation instrument for e-government web sites. In: INTERNET RESEARCH 7.0: INTERNET CONVERGENCES, 2006, Brisbane, Australia. Proceedings... Brisbane: Qut School of Information System, 2006.

HOLZER, M.; KIM, S.T. Digital governance in municipalities worldwide: a longitudinal assessment of municipal web sites throughout the world. Newark New Jersy: National Center for Public Productivity, 2005. Available from: <http:// unpan1.un.org/intradoc/groups/public/documents/aspa/ unpan022839.pdf>. Cited: 20 June 2009.

INTDEV Internet Technologies. Search engine optimization. 2007. Available from: <www.intedev.co.za>. Cited: 20 June 2009
JIMÉNEZ PIANO, M.; ORTIZ-REPISO JIMÉNEZ, V. Evaluación y calidad de sedes web. Gijón: Trea, 2007.

KUTLUCA, T.; AYDIN, S.; BAKI, A. Investigating web sites of faculties of education: the case of Turkey. The Turkish Online Journal of Educational Technology, v.8, n.2, p.82-90, 2009.

MERWE, R.; BEKKER, J. A framework and methodology for evaluating e-commerce web sites. Internet Research:Electronic Networking Applications and Policy, v.13, n.3, p.330-341, 2003.

MIRANDA GONZÁLEZ, F.; BAŃEGIL PALACIOS, T.M. Quantitative evaluation of commercial web sites: an empirical study of spanish firms. International Journal of Information Management, v.24, n.4, p.313-328, 2004.

NIELSEN, J. Designing web usability. Munich: Markt-Technik Verlag, 2004

OLSINA, L.; PAPA, F.; MOLINA, H. How to measure \& evaluate web applications in a consistent way. In: ROSSI, G. et al. (Ed.). Web engineering: modeling \& implementing web applications. London: Springer, 2008. p.385-420.

OPPENHEIM, C.; WARD, L. Evaluation of the web sites for B2C e-commerce. Aslib Proceedings, v.58, n.3, p.237-260, 2006.

PANOPOULOU, E.;TAMBOURIS, E.;TARABANIS, K. A framework for evaluationg web sites of public authorities. Aslib Proceedings, v.60, n.5, p.517-546, 2008.

SCHUBERT, P.; SELZ, D. Web assessment-measuring the efectiveness of electronic commerce sites going beyond traditional marketing paradigms. In: HAWAII INTERNATIONAL CONFERENCE ON SYSTEM SCIENCES, 32.,1999. Maui, Hawaii. Proceedings... Maui, Hawaii: IEEE Computer Society, 1999.

SMITH, A.G. Applying evaluation criteria to New Zealand government web sites. International Journal of Information Management, v.21, n.2, p.37-49, 2001.

TORO M. A model for building a better academic web site: a quantitative analysis of foreing language departments on the world wide web. 2002. Thesis (Master) - West Virginia University, Morgantown, 2002.

WANG, X.; LIU, J. Usability evaluation of B2C web site. In: INTERNATIONAL CONFERENCE ON WIRELESS COMMUNICATIONS, NETWORKING AND MOBILE COMPUTING, 3., 2007, Shanghai, Chine. Proceedings... Shanghai, Chine: IEEE, 2007. 
ANEXO 1

INDICADORES DE EVALUACIÓN DE LA CHECKLIST

\begin{tabular}{|c|c|c|c|c|}
\hline & 0 & 1 & 2 & 3 \\
\hline \multicolumn{5}{|l|}{ CRITERIOS DE CARÁCTER GENERAL } \\
\hline \multicolumn{5}{|l|}{ Identidad } \\
\hline \multicolumn{5}{|l|}{ Estar publicado bajo el dominio oficial de la universidad } \\
\hline \multicolumn{5}{|l|}{ URL corto y significativo con el contenido del sitio web y de cada página } \\
\hline \multicolumn{5}{|l|}{ Título corto y descriptivo que indique el nombre del programa de doctorado y de la universidad } \\
\hline \multicolumn{5}{|l|}{ Adecuados elementos de identidad visual } \\
\hline \multicolumn{5}{|l|}{ Nombre completo de la universidad, departamento y programa de doctorado en texto o en imagen } \\
\hline \multicolumn{5}{|l|}{ USABILIDAD Y ACCESIBILIDAD } \\
\hline \multicolumn{5}{|l|}{ Navegación } \\
\hline \multicolumn{5}{|l|}{ Enlace a la página web principal de la universidad } \\
\hline \multicolumn{5}{|l|}{ Identificación de los colaboradores del sitio web } \\
\hline \multicolumn{5}{|l|}{ Enlace al mapa del sitio web } \\
\hline \multicolumn{5}{|l|}{ Los elementos de navegación se presentan en la primera sección visual de las páginas del sitio web } \\
\hline \multicolumn{5}{|l|}{ El sitio web tiene minimizado su nivel de profundidad (de 3 a 5 niveles) para no complicar la navegación } \\
\hline \multicolumn{5}{|l|}{$\begin{array}{l}\text { El sitio web proporciona una ruta de navegación para que el Usuario ubique su posición dentro del sitio web y pueda } \\
\text { regresar utilizando esa ruta }\end{array}$} \\
\hline \multicolumn{5}{|l|}{ Consistencia en el formato de los enlaces para que sean fácilmente distinguibles } \\
\hline \multicolumn{5}{|l|}{ Bajos tiempos de acceso y de navegación entre secciones } \\
\hline \multicolumn{5}{|l|}{ Adecuada estructura lógica } \\
\hline \multicolumn{5}{|l|}{ Se indica el nombre de la sección donde se encuentra el usuario en cada página del sitio web } \\
\hline \multicolumn{5}{|l|}{ Correcta validación del sitio web (ausencia de "enlaces muertos") } \\
\hline \multicolumn{5}{|l|}{ Estilo gráfico } \\
\hline \multicolumn{5}{|l|}{ Aparece claramente la fecha de la última actualización } \\
\hline \multicolumn{5}{|l|}{ Vigencia de la información y eventos publicados } \\
\hline \multicolumn{5}{|l|}{ Apariencia del sitio web atractiva y elaborada de manera profesional } \\
\hline \multicolumn{5}{|l|}{ Distribución clara y consistente de las áreas que componen la pagina (navegación, menús, etc.) } \\
\hline \multicolumn{5}{|l|}{ Incluye páginas web construidas en base a las recomendaciones y especificaciones para HTML de la W3C } \\
\hline \multicolumn{5}{|l|}{ Presenta compatibilidad con diferentes versiones de navegadores } \\
\hline La imagen gráfica no afecta el funcionamiento del sitio web (no incrementa los tiempos de carga y recarga) & & & & \\
\hline Eequilibrada cantidad de imágenes & & & & \\
\hline Inclusión de fuentes tipográficas estándar para los navegadores & & & & \\
\hline $\begin{array}{l}\text { Acceso a información interna mediante intranets u otras herramientas con información sobre los estudios, material } \\
\text { docente, avisos, foros, tutorías online... }\end{array}$ & & & & \\
\hline Accesibilidad & & & & \\
\hline Presenta opciones para usuarios con necesidades especiales & & & & \\
\hline Visibilidad & & & & \\
\hline Incluye contador para el número de visitas & & & & \\
\hline Uso de metaetiquetas (con información sobre el contenido e imágenes del sitio web página, palabras clave etc.) & & & & \\
\hline INFORMACIÓN Y CONTENIDOS & & & & \\
\hline investigación & & & & \\
\hline Se indican claramente las líneas de investigación del programa de & & & & \\
\hline
\end{tabular}


ANEXO 1

INDICADORES DE EVALUACIÓN DE LA CHECKLIST

\begin{tabular}{|c|c|c|c|}
\hline & 0 & $1 \quad 2$ & 3 \\
\hline Se indican claramente los criterios seguidos para la dirección de tesis doctorales & & & \\
\hline Aparece la relación de profesores e investigadores encargados de su dirección & & & \\
\hline Requisitos y resultados & & & \\
\hline Se indica claramente el perfil de ingreso & & & \\
\hline Se indican claramente los criterios de admisión & & & \\
\hline Se indican claramente los criterios de reconocimiento y convalidación de formación previa & & & \\
\hline Se indican claramente los resultados académicos obtenidos en el programa de postgrado & & & \\
\hline Se incluye información y estudios sobre la inserción laboral de los titulados & & & \\
\hline Claridad de la información & & & \\
\hline Uso de un lenguaje simple, claro y directo & & & \\
\hline $\begin{array}{l}\text { Uso de palabras apropiadas teniendo en cuenta el contenido que se ofrece y el perfil de la comunidad a la que se dirige } \\
\text { la información }\end{array}$ & & & \\
\hline Estructura gramatical, ortografía y redacción correctas & & & \\
\hline Traducción clara y exacta de términos en idiomas diferentes al español & & & \\
\hline Contenidos & & & \\
\hline Se indican claramente los objetivos generales del programa de postgrado & & & \\
\hline Se indican claramente los objetivos específicos de cada curso de postgrado & & & \\
\hline Se indica claramente la programación académica & & & \\
\hline Se indican claramente los créditos correspondientes a cada curso & & & \\
\hline Se indica claramente el contenido explícito de los cursos & & & \\
\hline Se indica claramente la metodología y el aprendizaje desarrollados en cada curso & & & \\
\hline Se indican claramente los criterios y procedimientos de evaluación de cada curso & & & \\
\hline Se indican claramente la bibliografía recomendada y utilizada para cada curso & & & \\
\hline Aparecen tablones de anuncios con novedades & & & \\
\hline Formularios/test valoración & & & \\
\hline Se incluyen encuestas generales sobre el postgrado & & & \\
\hline Se incluyen encuestas de egresados & & & \\
\hline Se incluyen formularios de seguimiento & & & \\
\hline SERVICIOS & & & \\
\hline Motor de búsqueda & & & \\
\hline El sitio web incluye un motor de búsqueda interno que permite plantear búsquedas por palabras & & & \\
\hline Los resultados ofrecidos por el motor de búsqueda aparecen en listados paginados & & & \\
\hline $\begin{array}{l}\text { Los resultados ofrecidos por el motor de búsqueda proveen información precisa respecto a la página que contiene las } \\
\text { palabras clave de la consulta }\end{array}$ & & & \\
\hline Los resultados ofrecidos por el motor de búsqueda incluyen un hiperenlace a la información de interés & & & \\
\hline En la lista de resultados ofrecidos por el motor de búsqueda se resaltan las palabras clave de la consulta & & & \\
\hline Contactos y accesos & & & \\
\hline Se incluye una opción para sugerencias y quejas & & & \\
\hline Se incluye correo-e de contacto para asesoría, dudas, solicitudes, comentarios sobre el sitio web y su contenido etc. & & & \\
\hline Se incluye dirección postal y teléfono del departamento y/o centro que organiza el programa & & & \\
\hline $\begin{array}{l}\text { Bajos tiempos de respuesta a las consultas planteadas por los usuarios sobre diferentes aspectos del programa de } \\
\text { postgrado }\end{array}$ & & & \\
\hline
\end{tabular}


ANEXO 2

ANÁLISIS DE LOS COMPONENTES PRINCIPALES Y SU CORRESPONDENCIA CON LOS CLUSTERS

La siguiente tabla compara los resultados de la aplicación de las técnicas de análisis de clustery del análisis de componentes principales.

\begin{tabular}{|c|c|c|c|c|r|r|}
\hline \multicolumn{3}{|c|}{ Componentes } & \multicolumn{3}{c|}{ Cluster } & 皇 \\
\hline 1 & 2 & 3 & 1 & 2 & 3 & Promedio \\
\hline
\end{tabular}

Máxima adscripción al componente 1

\begin{tabular}{|l|l|l|l|l|l|l|}
\hline 0.84 & 0.24 & 0.07 & & $x$ & 1.67 \\
\hline 0.81 & 0.26 & 0.13 & & & $x$ & 1.79 \\
\hline 0.80 & 0.09 & 0.23 & & & $x$ & 1.59 \\
\hline 0.78 & 0.22 & 0.14 & & & $x$ \\
\hline
\end{tabular}

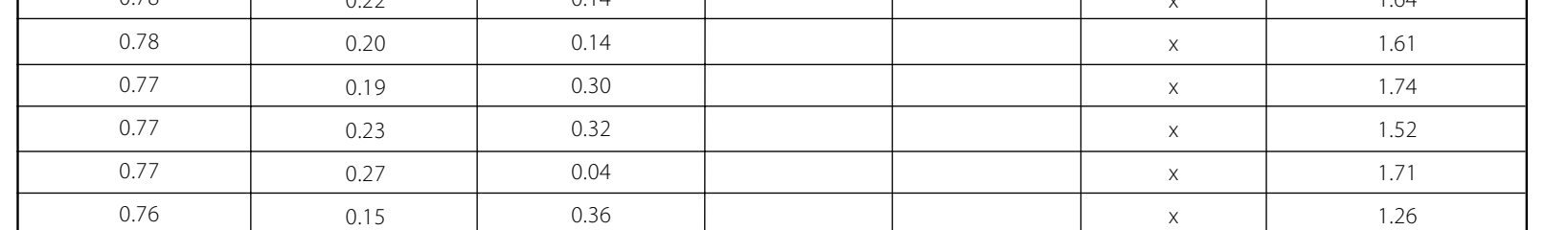

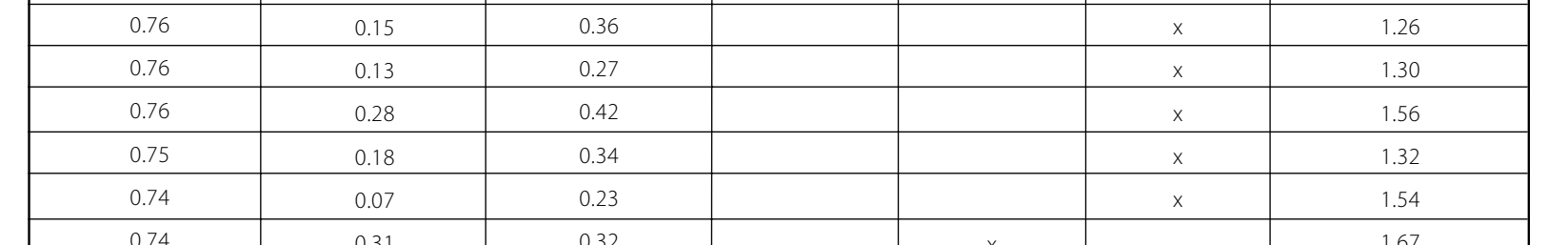

\begin{tabular}{|c|c|c|c|c|c|}
\hline 0.14 & 0.07 & 0.23 & & $x$ & 1.54 \\
\hline 0.74 & 0.31 & 0.32 & $x$ & & 1.67 \\
\hline 0.74 & 0.16 & 0.14 & & $x$ & 1.55 \\
\hline 0.73 & 0.22 & 0.28 & & $x$ & 118 \\
\hline 0.73 & 0.34 & 0.24 & & $x$ & 1.66 \\
\hline 0.72 & 0.25 & 0.16 & & $x$ & 1.66 \\
\hline 0.72 & 0.34 & 0.37 & & $x$ & 1.71 \\
\hline 0.72 & 0.31 & 0.22 & & $x$ & 1.96 \\
\hline 0.71 & 0.13 & 0.19 & & $x$ & 1.77 \\
\hline 0.71 & 0.27 & 0.40 & & $x$ & 1.47 \\
\hline 0.71 & 0.32 & 0.23 & & $x$ & 1.61 \\
\hline 0.70 & 0.12 & 0.39 & & $x$ & 1.33 \\
\hline 0.70 & 0.33 & 0.24 & & $x$ & 1.62 \\
\hline 0.69 & 0.19 & 0.32 & & $x$ & 1.41 \\
\hline 0.69 & 0.31 & 0.20 & & $x$ & 1.76 \\
\hline 0.69 & 0.06 & 0.19 & & $x$ & 1.65 \\
\hline 0.69 & 0.42 & 0.34 & & $x$ & 1.87 \\
\hline 0.67 & 0.30 & 0.37 & & $x$ & 1.89 \\
\hline 0.66 & 0.22 & 0.52 & $x$ & & 1.34 \\
\hline 0.66 & 0.12 & 0.01 & & $x$ & 2.05 \\
\hline 0.65 & 0.20 & 0.11 & & $x$ & 1.76 \\
\hline 0.65 & 0.28 & 0.41 & & $x$ & 1.81 \\
\hline 0.64 & 0.02 & 0.27 & & $x$ & 1.76 \\
\hline 0.64 & 0.19 & 0.30 & & $x$ & 1.40 \\
\hline 0.64 & 0.29 & 0.32 & & $x$ & 1.36 \\
\hline 0.64 & 0.25 & 0.08 & & $x$ & 1.75 \\
\hline 0.64 & 0.13 & 0.06 & & $x$ & 2.16 \\
\hline
\end{tabular}


ANEXO 2

ANÁLISIS DE LOS COMPONENTES PRINCIPALES Y SU CORRESPONDENCIA CON LOS CLUSTERS

La siguiente tabla compara los resultados de la aplicación de las técnicas de análisis de clustery del análisis de componentes principales.

Continuación

\begin{tabular}{|c|c|c|c|c|c|c|}
\hline \multicolumn{3}{|c|}{ Componentes } & \multicolumn{3}{|c|}{ Cluster } & \multirow[b]{2}{*}{ Promedio } \\
\hline 1 & 2 & 3 & 1 & 2 & 3 & \\
\hline \multicolumn{7}{|c|}{ Máxima adscripción al componente 1} \\
\hline 0.63 & 0.25 & 0.16 & & & $x$ & 1.99 \\
\hline 0.61 & 0.04 & 0.20 & & & $x$ & 1.67 \\
\hline 0.61 & 0.15 & 0.45 & & & $x$ & 1.31 \\
\hline 0.61 & 0.06 & 0.30 & & & $x$ & 1.68 \\
\hline 0.61 & 0.37 & 0.39 & & & $x$ & 1.79 \\
\hline 0.60 & 0.35 & 0.55 & & & $x$ & 1.73 \\
\hline 0.60 & 0.28 & 0.50 & & $x$ & & 1.58 \\
\hline 0.59 & 0.33 & 0.38 & & & $x$ & 1.57 \\
\hline 0.59 & 0.36 & 0.43 & & & $x$ & 1.91 \\
\hline 0.59 & 0.22 & 0.57 & & $x$ & & 1.32 \\
\hline 0.57 & 0.56 & 0.10 & & & $x$ & 1.93 \\
\hline 0.57 & 0.31 & 0.45 & & & $x$ & 2.00 \\
\hline 0.55 & 0.40 & 0.52 & & $x$ & & 1.36 \\
\hline 0.52 & 0.26 & 0.24 & & & $x$ & 2.24 \\
\hline 0.51 & 0.38 & 0.20 & $x$ & & & 1.54 \\
\hline 0.50 & 0.34 & 0.34 & $x$ & & & 1.08 \\
\hline 0.49 & 0.39 & 0.16 & & & $x$ & 1.41 \\
\hline 0.48 & 0.15 & 0.18 & & & $x$ & 2.04 \\
\hline
\end{tabular}

Máxima adscripción al componente 2

58

\begin{tabular}{|l|l|l|l|l|l|l|}
\hline 0.20 & 0.84 & 0.25 & $x$ & & 1.35 \\
\hline 0.21 & 0.82 & 0.22 & $x$ & & & 1.28 \\
\hline 0.21 & 0.82 & 0.22 & $x$ & & & 1.28 \\
\hline 0.29 & 0.82 & 0.15 & $x$ & & & 1.67 \\
\hline 0.16 & 0.81 & 0.21 & $x$ & & & 1.49 \\
\hline 0.33 & 0.79 & -0.08 & $x$ & & & 1.51 \\
\hline 0.24 & 0.79 & 0.22 & $x$ & & & 1.32 \\
\hline 0.17 & 0.79 & 0.18 & $x$ & & & 1.55 \\
\hline 0.17 & 0.79 & 0.33 & $x$ & & & 1.51 \\
\hline 0.13 & 0.77 & 0.30 & $x$ & & & 1.56 \\
\hline 0.04 & 0.76 & 0.53 & $x$ & & & 1.12 \\
\hline 0.05 & 0.76 & 0.39 & $x$ & & & 1.17 \\
\hline 0.24 & 0.75 & 0.12 & $x$ & & & 1.83 \\
\hline 0.33 & 0.75 & 0.35 & $x$ & & & 1.50 \\
\hline 0.12 & 0.74 & 0.50 & $x$ & & & 1.10 \\
\hline 0.26 & 0.73 & 0.07 & $x$ & & & 1.29 \\
\hline 0.21 & 0.72 & 0.22 & $x$ & & & 1.37 \\
\hline 0.16 & 0.72 & 0.14 & $x$ & & & 1.69 \\
\hline 0.38 & 0.72 & 0.18 & $x$ & & & 1.46 \\
\hline 0.24 & 0.69 & 0.20 & $x$ & & & 1.13 \\
\hline
\end{tabular}

TransInformação, Campinas, 24(1):47-60, jan./abr., 2012 
ANEXO 2

ANÁLISIS DE LOS COMPONENTES PRINCIPALES Y SU CORRESPONDENCIA CON LOS CLUSTERS

La siguiente tabla compara los resultados de la aplicación de las técnicas de análisis de cluster y del análisis de componentes principales.

Continuación

\begin{tabular}{|c|c|c|c|c|c|c|}
\hline \multicolumn{3}{|c|}{ Componentes } & \multicolumn{3}{|c|}{ Cluster } & \multirow[b]{2}{*}{ Promedio } \\
\hline 1 & 2 & 3 & 1 & 2 & 3 & \\
\hline
\end{tabular}

Máxima adscripción al componente 2

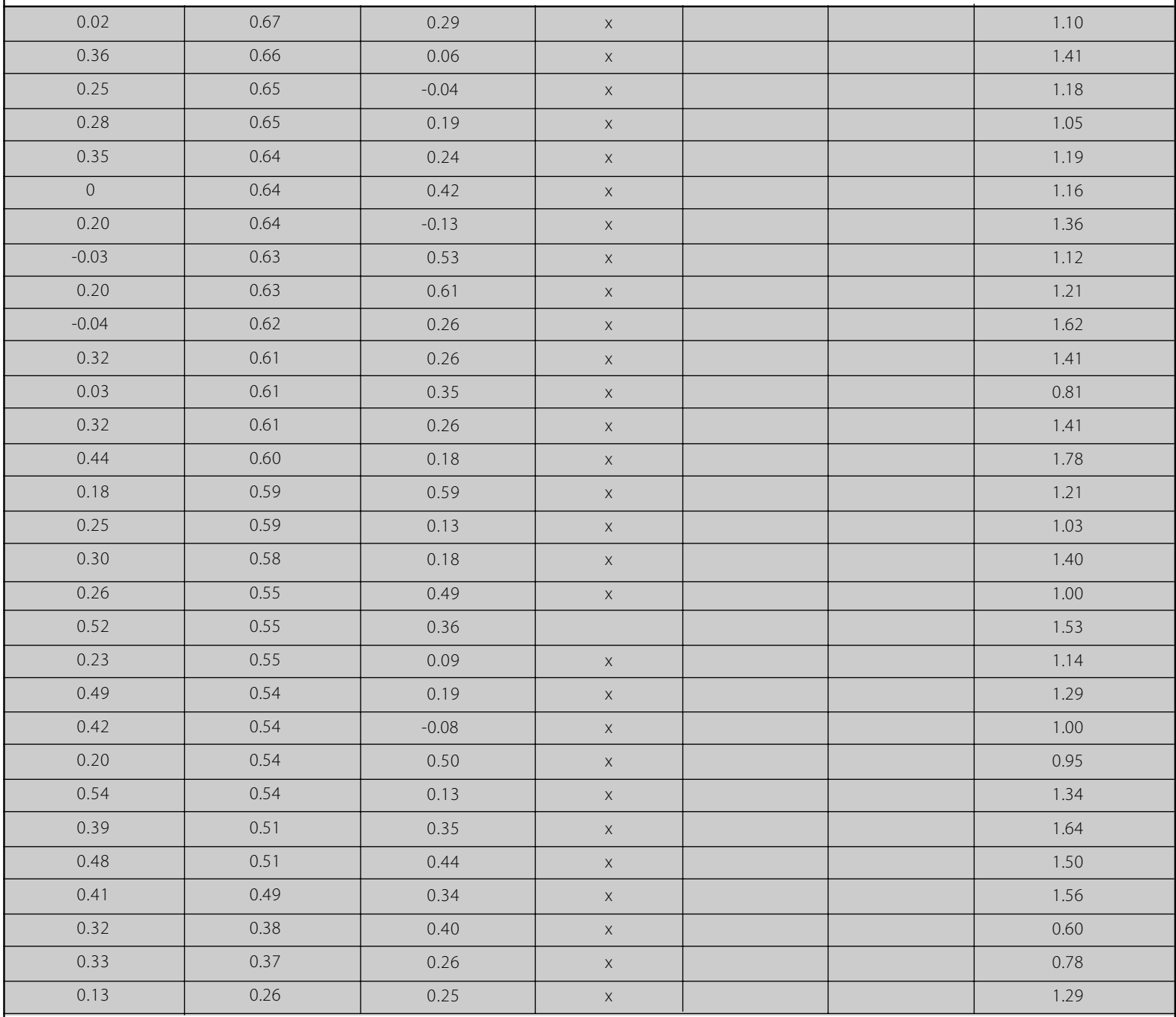

Máxima adscripción al componente 3

\begin{tabular}{|l|l|l|l|l|l|l|}
\hline 0.41 & 0.20 & 0.82 & & $x$ & $x$ & 1.44 \\
\hline 0.39 & 0.20 & 0.82 & 0.80 & & $x$ & 1.50 \\
\hline 0.32 & 0.30 & 0.80 & & $x$ & 1.48 \\
\hline 0.34 & 0.20 & 0.80 & & $x$ & 1.51 \\
\hline 0.37 & 0.10 & 0.77 & & $x$ & 1.38 \\
\hline 0.41 & 0.20 & 0.76 & & $x$ & 1.49 \\
\hline 0.43 & 0.20 & 0.74 & & 1.45 \\
\hline 0.44 & 0.20 & & & 1.45 \\
\hline
\end{tabular}


ANEXO 2

ANÁLISIS DE LOS COMPONENTES PRINCIPALES Y SU CORRESPONDENCIA CON LOS CLUSTERS

La siguiente tabla compara los resultados de la aplicación de las técnicas de análisis de clustery del análisis de componentes principales.

Conclusión

\begin{tabular}{|c|c|c|c|c|c|c|}
\hline \multicolumn{3}{|c|}{ Componentes } & \multicolumn{3}{|c|}{ Cluster } & \multirow[b]{2}{*}{ Promedio } \\
\hline 1 & 2 & 3 & 1 & 2 & 3 & \\
\hline \multicolumn{7}{|c|}{ Máxima adscripción al componente 3} \\
\hline 0.46 & 0.30 & 0.74 & & $x$ & & 1.45 \\
\hline 0.41 & 0.10 & 0.73 & & $x$ & & 1.60 \\
\hline 0.49 & 0.20 & 0.72 & & $x$ & & 1.42 \\
\hline 0.50 & 0.30 & 0.69 & & $x$ & & 1.31 \\
\hline 0.54 & 0.20 & 0.65 & & $x$ & & 1.28 \\
\hline 0.57 & 0.20 & 0.64 & & $x$ & & 1.39 \\
\hline 0.51 & 0.20 & 0.64 & & $x$ & & 1.52 \\
\hline 0.51 & 0.20 & 0.63 & & $x$ & & 1.15 \\
\hline 0.54 & 0.20 & 0.62 & & $x$ & & 1.45 \\
\hline 0.54 & 0.20 & 0.62 & & $x$ & & 1.47 \\
\hline 0.37 & 0.40 & 0.60 & $x$ & & & 1.11 \\
\hline 0.08 & 0.40 & 0.53 & $x$ & & & 0.90 \\
\hline 0.11 & 0.40 & 0.50 & $x$ & & & 0.88 \\
\hline 0.31 & 0.10 & 0.49 & & & $x$ & 1.87 \\
\hline 0.03 & 0.40 & 0.48 & $x$ & & & 0.91 \\
\hline 0.33 & 0.40 & 0.48 & $x$ & & & 0.72 \\
\hline
\end{tabular}

